\title{
Dermatomyositis Revealing Breast and Ovarian Malignancies: Report of a Case
}

\section{Eman Almohawes*}

Department of Diagnostic Radiology, Security Forces Hospital King Fahd Suburb, KSA

*Corresponding author: Almohawes E, Consultant and Head of Department of Diagnostic Radiology, Security forces Hospital King Fahd Suburb, 5171, Dammam, KSA

Received: December 06, 2017; Accepted: J anuary 05, 2018; Published: J anuary 31, 2018

\section{Abbreviation}

DM: Dermatomyositis; MLO: Mediolateral Oblique; CC: CranioCaudal; CT: Computed Tomography; US: Ultrasonography

\section{Introduction}

Dermatomyositis is rare type of the idiopathic inflammatory myopathies which clinically presents with progressive symmetrical proximal muscle weakness and a characteristic rash. However, this disease primarily involves the skin and the muscles, it is considered as systemic disease with manifestations involving other body systems. Dermatomyositis is linked to internal malignancy with incidence between $15 \%$ and $25 \%$ [1]. It is rare condition in the literature, we report an additional case of dermatomyositis associated with breast and ovarian malignancies.

\section{Patient and observation}

A 51-year-old female patient, known case of Dermatomyositis. The story started when the patient realizes that there is abnormal lump and hardness in her right breast at the site of nipple and areola with abnormal inversion of the nipple, she sought medical advice and upon examination: the physician discovered hard immobile retroareolar mass in the right breast and this mass was suspicious for him and causing nipple retraction so he requested mammography for the patient for further evaluation of this mass. Mammogram done and revealed retracted nipple with retroareolar coarse heterogeneous calcification and underlying ill-defined mass with speculation (Figure 1), after that complementary US done with elastography and revealed: irregular hypoechoic nodule at retroareolar region with hyperechoic rim indicating desmoplastic reaction, appear hard on elastography. Right axillary lymphnodes show focal cortical bulge with increased vascularity (Figure 2). After that core biopsy done from the breast mass (Figure 3) and from the right axillary lymphnode and the histopathology came as Invasive Ductal Carcinoma, after that CT scan of the chest, abdomen and pelvis requested for staging which revealed right ovarian neoplastic mass as well as redemonstration of right breast mass with calcification (Figure 4).

\section{Discussion}

Dermatomyositis is a rare heterogeneous connective tissue diseasewhich characterized by aninflammatory process involving the

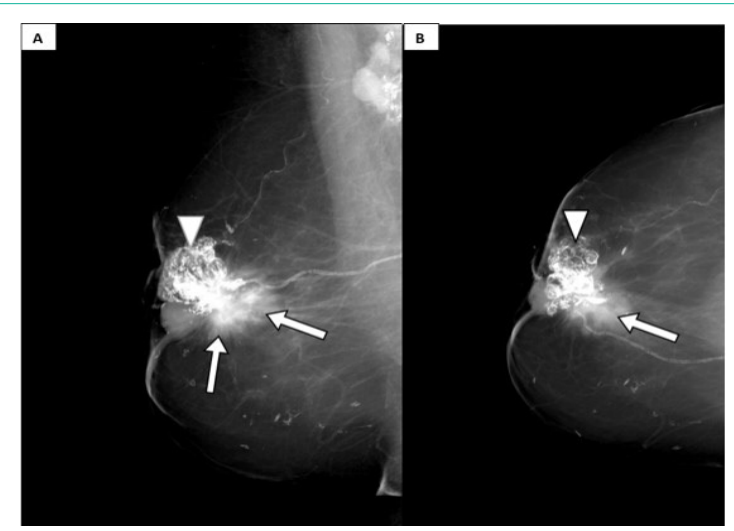

Figure 1: Right breast mammogram (A: MLO view B: CC view) revealed retracted right nipple with retroareolar coarse heterogeneous calcification (arrow head) with underlying ill-defined mass with speculation (arrow).
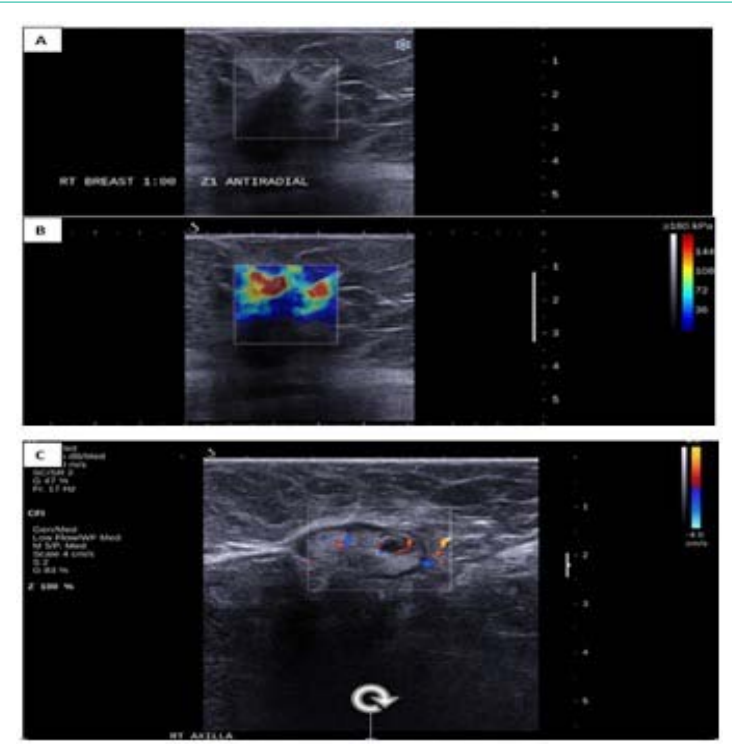

Figure 2: Ultrasound examination (A) showed: irregular hypoechoic nodule at 1:00 retroareolar with hyperechoic rim indicating desmoplastic reaction, appear hard on elastography (B). Right axillary ultrasound reveals lymphnode with focal cortical bulge and increased vascularity (C). 

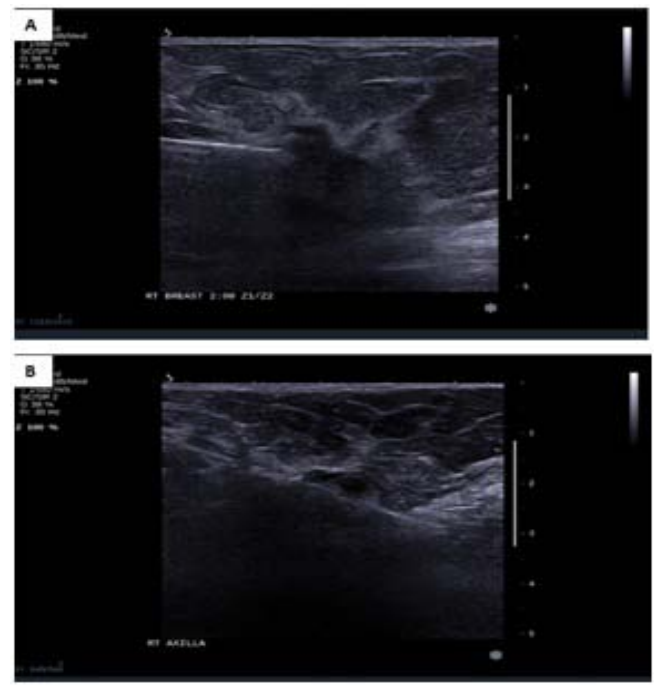

Figure 3: Ultrasound guided biopsy from the mass $(A)$ and from the right axillary lymphnodes (B).
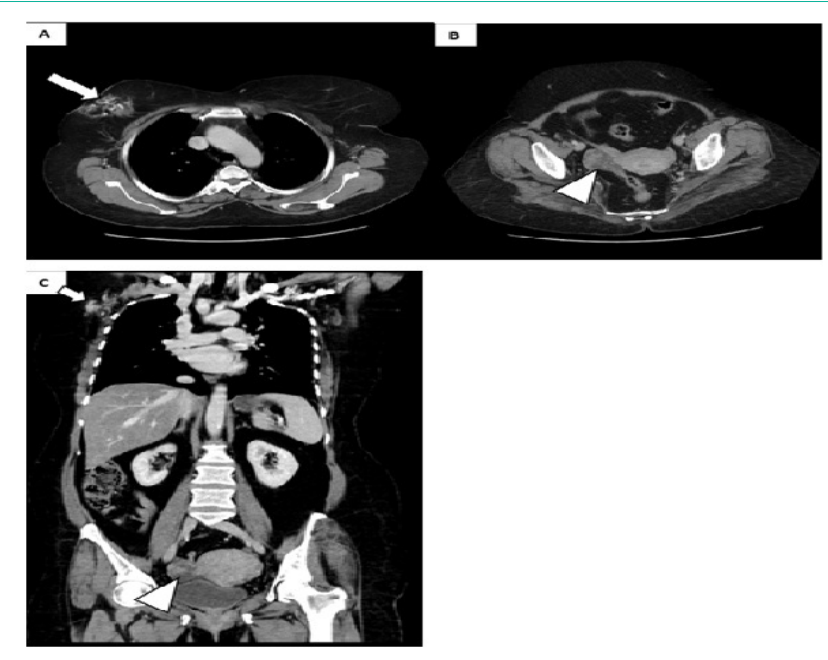

Figure 4: Selected axial cuts at the level of the aortic arch (A) and at pelvic region (B) as well as coronal reformate reveals right breast mass with calcification (arrow) and right ovarian heterogeneous mass (arrowhead).

skin, skeletal muscles, and other connective tissues [2]. In juvenile dermatomyositis, soft tissue calcifications termed "calcinosis"are common in approximately $10 \%-40 \%$, however it is uncommon in cases of adult onset dermatomyositis [3]. Risk factors attributed to the development of calcinosisinclude young age and delayed diagnosis ortherapy [4].

The incidence of dermatomyositis is approximately $1 / 100,000$. It can affect both children and adults with female to male ratio of $(2: 1)$. Clinically, the main manifestations are proximal muscle weakness and skin rash.

It has been reported that dermatomyositis carries an increased risk of malignancy and also can present as a paraneoplastic syndrome to multiple types of underlying malignancies [5]. A study from Malaysia by Tang and The varajah in 2010 resulted in $47.4 \%$ of dermatomyositis patients had underlying malignancy [6].
The relationship between presence of dermatomyositis and cancer is not yet fully understood. One theory refers to that the antigen present in muscle in cases of myositis are the same that expressed in any various types of cancers associated with inflammatory myopathies, hence the relationship generated, so the link between cancer and dermatomyositis to be an expression of antigens common to the cancer and to muscle tissue in some patients with dermatomyositis [7].

The onset relationship between Paraneoplastic dermatomyositis and diagnosis of cancer is variable as it may be able to precede, coincide with, or develop after. Elderly male with dermatomyositis are the most common group associated with high risk of cancer. In female the most common cancers associated with adult dermatomyositis are breast and ovary.

Reviewing the studies from literature regarding the type of cancers associated with dermatomyositis we found it different from region to another, for example in Europe, the cancers associated with adult dermatomyositis include, in order of frequency, ovary, lung, breast, colon and rectum, stomach, and pancreas [8]. Other associated cancers include prostate and non-Hodgkin lymphoma.

Sweden, Denmark and Finland Hill, et al. reported that the most common types of cancer are ovarian, lung and pancreatic cancers, however in Scotland, lungcancer was the most common cancer related to dermatomyositis, while in Tunisia it was breast cancer.

Regarding the management of paraneoplastic dermatomyositis, the main issue is to control the underlying neoplasm.

\section{Conclusion}

Dermatomyositis is a systemic connective tissue disease involving mainly the skin but can affect various connective tissue in the body systems with known association with types of cancers mainly the breast and gynecological malignancies in female and other types in male with different studies reported different types and here it will be part of paraneoplastic syndrome where it called paraneoplastic dermatomyositis.

\section{References}

1. Callen JP, Wortmann RL. Dermatomyositis. Clinics in Dermatology. 2006; 24: 363-373.

2. Dalakas MC. Inflammatory Muscle Diseases. N Engl J Med. 2015; 373: 393394.

3. Callen JP. Dermatomyositis. The Lancet. 2000; 53-57.

4. Weinel S, Callen JP. Calcinosis cutis complicating adult-onset dermatomyositis. Arch Dermatol. 2004; 140: 365-366.

5. Lamquami S, Errarhay S, Mamouni N, Bouchikhi C, Banani A. Dermatomyositis revealing breast cancer: report of a case. Pan Afr Med J. 2015; 21: 89.

6. Tang MM, Thevarajah S. Paraneoplastic Dermatomyositis: A 12-year Retrospective Review in the Department of Dermatology Hospital Kuala Lumpur. Med J Malaysia. 2010; 65:138-142.

7. Requena C, Alfaro A, Traves V, Nagore E, Llombart B, Serra C, et al. Paraneoplastic Dermatomyositis: a study of 12 cases. Actas Dermosifiliogr. 2014; 105: 675-682.

8. Teoh JW, Yunus RM, Hassan F, Ghazali N, Abidin ZA. Nasopharyngeal carcinoma in dermatomyositis patients: a 10- year retrospective review in Hospital Selayang, Malaysia. Rep Pract Oncol Radiother. 2014; 19: 332-336. 